\title{
Genetic models reveal historical patterns of sea lamprey population fluctuations within Lake Champlain
}

Cassidy C D'Aloia, Christina B Azodi, Sallie P Sheldon, Stephen C Trombulak, Willam R Ardren

The origin of sea lamprey (Petromyzon marinus) in Lake Champlain has been heavily debated over the past decade. Given the lack of historical documentation, two competing hypotheses have emerged in the literature. First, it has been argued that the relatively recent population size increase and concomitant rise in wounding rates on prey populations are indicative of an invasive population that entered the lake through the Champlain Canal. Second, recent genetic evidence suggests a post-glacial colonization at the end of the Pleistocene, approximately 11,000 years ago. One limitation to resolving the origin of sea lamprey in Lake Champlain is a lack of historical and current measures of population size. In this study, the issue of population size was explicitly addressed using nuclear (nDNA) and mitochondrial DNA (mtDNA) markers to estimate historical demography with genetic models. Haplotype network analysis, mismatch analysis, and summary statistics based on mtDNA noncoding sequences for $\mathrm{NCl}$ (479 bp) and $\mathrm{NCll}$ (173 bp) all indicate a recent population expansion. Coalescent models based on mtDNA and nDNA identified two potential demographic events: a population decline followed by a very recent population expansion. The decline in effective population size may correlate with land-use and fishing pressure changes post-European settlement, while the recent expansion may be associated with the implementation of the salmonid stocking program in the 1970s. These results are most consistent with the hypothesis that sea lamprey are native to Lake Champlain; however, the credibility intervals around parameter estimates demonstrate that there is uncertainty regarding the magnitude and timing of past demographic events. 


\section{TITLE:}

2 Genetic models reveal historical patterns of sea lamprey population fluctuations

3 within Lake Champlain

4

5 Authors:

6 1. Cassidy C. D’Aloia*; Middlebury College, Department of Biology, Middlebury, VT, USA

7 Current Affiliation: University of Toronto, Department of Ecology \& Evolutionary Biology,

8 Toronto, ON, CA

10 2. Christina B. Azodi; Middlebury College, Department of Molecular Biology and Biochemistry,

11 Middlebury, VT, USA

12 Current Affiliation: Michigan State University, Department of Plant Biology, East Lansing, MI, 13 USA

3. Sallie P. Sheldon; Middlebury College, Department of Biology, Middlebury, VT, USA

4. Stephen C. Trombulak; Middlebury College, Department of Biology and Program in Environmental Studies, Middlebury, VT, USA

5. William R. Ardren; U.S. Fish and Wildlife Service, Western New England Complex, Essex 21 Junction, VT, USA

$23 *$ Corresponding Author:

24 Current contact information:

25 Cassidy C. D’Aloia; University of Toronto, Department of Ecology \& Evolutionary Biology, 25

26 Harbord St, Toronto, ON M5S 3G5, Canada; Phone: (647) 939-7722;

27 cassidy.daloia@utoronto.ca 


\section{ABSTRACT}

32 The origin of sea lamprey (Petromyzon marinus) in Lake Champlain has been heavily debated over the past decade. Given the lack of historical documentation, two competing hypotheses have emerged in the literature. First, it has been argued that the relatively recent population size increase and concomitant rise in wounding rates on prey populations are indicative of an invasive population that entered the lake through the Champlain Canal. Second, recent genetic evidence suggests a post-glacial colonization at the end of the Pleistocene, approximately 11,000 years ago. One limitation to resolving the origin of sea lamprey in Lake Champlain is a lack of historical and current measures of population size. In this study, the issue of population size was explicitly addressed using nuclear (nDNA) and mitochondrial DNA (mtDNA) markers to estimate historical demography with genetic models. Haplotype network analysis, mismatch analysis, and summary statistics based on mtDNA noncoding sequences for NCI (479 bp) and NCII (173 bp) all indicate a recent population expansion. Coalescent models based on mtDNA and nDNA identified two potential demographic events: a population decline followed by a very recent population expansion. The decline in effective population size may correlate with land-use and fishing pressure changes post-European settlement, while the recent expansion may be associated with the implementation of the salmonid stocking program in the 1970s. These results are most consistent with the hypothesis that sea lamprey are native to Lake Champlain; however, the credibility intervals around parameter estimates demonstrate that there is uncertainty regarding the magnitude and timing of past demographic events. 


\section{INTRODUCTION}

55 The origin of the landlocked population of sea lamprey (Petromyzon marinus) in Lake

56 Champlain has been the subject of an ongoing debate in recent years (Bryan et al., 2005;

57 Waldman, Grunwald \& Wirgin, 2006; Waldman et al., 2009; Eshenroder, 2009, 2014). The sea

58 lamprey is an anadromous fish that has a parasitic juvenile phase during which it feeds on the

59 bodily fluids of a variety of prey fishes, including large salmonids such as lake trout (Salvelinus

60 namaycush), Atlantic salmon (Salmo salar), and lake whitefish (Coregonus clupeaformis), as

61 well as lake sturgeon (Acipenser fulvescens). Recent research has suggested that native coastal

62 populations of sea lamprey may have positive environmental impacts on freshwater streams. For

63 example, post-spawning sea lamprey carcasses may be important sources of marine-derived

64 nutrients and materials in oligotrophic streams (Guyette et al., 2014). However, the overall effect

65 of landlocked sea lamprey populations in the Great Lakes and Lake Champlain has been

66 detrimental. Lamprey-induced collapses of native fish populations have been well documented in

67 the region since the 1970s (Smith, 1971; Smith \& Tibbles, 1980).

68 Consequently, control efforts were developed in the Great Lakes to suppress sea lamprey

69 populations and facilitate restoration of native species. Control methods are varied and include

70 widespread biocide use, physical migration barriers, and spawning-phase traps in tributaries.

71 Although these methods have been criticized for the potential negative effects that non-target

72 species may experience (McLaughlin, Marsden \& Hayes, 2003), the effort has been largely

73 successful in terms of lamprey control. Using the methods developed in the Great Lakes, an

74 experimental lamprey control program was implemented in Lake Champlain in 1990, followed

75 by a long-term program beginning in 2001. Although the control methods in Lake Champlain are

76 similar to those in the Great Lakes, a major difference is the consensus regarding the fish's status 
77 as native versus invasive; in the Great Lakes, sea lamprey are known to be invasive (with the exception of Lake Ontario), while much debate surrounds the population in Lake Champlain. To date, two alternative historical scenarios have dominated the discussion regarding the origin of sea lamprey in Lake Champlain. First, it has been argued that individuals from the Atlantic coast population invaded Lake Champlain via the canal system sometime between the 1840s and the 1920s (Eshenroder, 2009, 2014). This hypothesis is based on the fact that the first documentation of the species in the lake was in 1929 (Eshenroder, 2014), 13 years after the construction of the Champlain Barge Canal (the third version of the Champlain Canal), which directly connected Lake Champlain to the Hudson River. Second, recent genetic analyses based on both nuclear (nDNA) and mitochondrial DNA (mtDNA) markers suggest that sea lamprey are native to Lake Champlain (Bryan et al., 2005; Waldman, Grunwald \& Wirgin, 2006; Waldman et al., 2009). Bryan et al. (2005) tested alternative coalescent-based colonization models using microsatellite markers and found evidence of long-term vicariance in the Lake Champlain population, concluding that the most probable route of entry was via the St. Lawrence River upon the initial formation of Lake Champlain about 12,500 years ago. Waldman et al. (2006) compared haplotype frequencies of the Lake Champlain population to Atlantic Coast and Great Lakes populations using mitochondrial non-coding DNA and concluded that the data were most consistent with a post-glacial colonization during a period when modern-day Lake Champlain was an arm of the Atlantic Ocean called the Champlain Sea. However, neither hypothesis has been widely accepted throughout the scientific and management communities. The "nonnative" hypothesis has been criticized because it is based on the absence of species documentation data during the $1800 \mathrm{~s}$ - a time when systematic biological censuses were not conducted in the lake - and because it lacks due consideration of the post-glacial geological history of the region. 
100 Likewise, the "native" hypothesis has recently been called into question after an extensive

101 review of historical documentation suggested that the time of origin used in genetic models

102 (1841) was based on an erroneous species identification and, subsequently, may have biased the

103 results (Eshenroder, 2014).

104 As a result of this ongoing debate, prior research has focused exclusively on the timing of

105 the origin of $P$. marinus in Lake Champlain, while little is known about the rest of the

106 population's history. The two previous genetic studies of $P$. marinus in the lake were regional

107 studies that compared the Lake Champlain sea lamprey population to other populations from the

108 Great Lakes and the Atlantic Ocean (Bryan et al., 2005; Waldman, Grunwald \& Wirgin, 2006).

109 While both studies found evidence for long-term vicariance from anadramous and other

110 freshwater populations, it has proven challenging to reconcile the results with the complete lack

111 of historical documentation of sea lamprey in the Lake Champlain (Bryan et al., 2005). Given

112 that these regional studies have already shown the Lake Champlain population to be

113 differentiated from all other populations, additional analyses can further probe the genetic data

114 by conducting rigorous intra-population analyses to model historical population dynamics within

115 Lake Champlain. An objective assessment of the timing and magnitude of fluctuations in

116 population size over time may therefore provide a more complete understanding of the history of

117 P. marinus in Lake Champlain, while simultaneously shedding new light on the contentious

118 topic of whether or not the species is invasive.

119 In cases such as this one, where historical census data are unavailable, genetic markers

120 can be powerful tools for inferring demographic fluctuations. These inferences are possible

121 because the census population size $\left(\mathrm{N}_{\mathrm{c}}\right)$ is generally proportional to the effective population size

$122\left(\mathrm{~N}_{\mathrm{e}}\right)$. Effective population size is the size of an idealized population (i.e., with binomial variance 
123 in reproductive success, an equal sex ratio, and discrete generations), that is subject to the same

124 level of genetic drift and inbreeding as the census population (Wright, 1938). Drawing on the

125 relationship between effective and census population size, and the fact that rapid demographic

126 fluctuations can be detected with genetic markers, we can investigate how effective population

127 size has changed over time and, in turn, infer proportional changes in the overall population

128 (Waples, 1989; Frankham, 1995).

129 Traditionally, genetic approaches to estimating historical demography have used

130 summary statistics to test whether extant population-level data deviate from theoretical

131 expectations under alternative models of population stasis, contractions, and expansions (Cornuet

132 \& Luikart, 1996; Harpending et al., 1998; Schneider \& Excoffier, 1999; Garza \& Williamson,

133 2001). For example, mismatch analysis uses sequence data to compare the distribution of

134 observed pairwise differences between all haplotypes in a population to the distribution expected

135 under a specified population change. Expansion, contractions, and equilibrium each generate a

136 particular pattern of the distribution of pairwise differences among sequences. Contractions or

137 equilibrium lead to multimodal, ragged distributions while expansions result in a smooth

138 unimodal Poisson distribution of pairwise differences (Harpending et al., 1998; Schneider \&

139 Excoffier, 1999). These moment-based metrics are widely used because they are easy to obtain

140 with sequence and/or allele frequency data. However, they provide crude approximations of

141 population changes, and their precision is linked to the timing and magnitude of the demographic

142 change in question.

143 Arguably, a more powerful approach to inferring past demographic change is coalescent

144 modeling (Storz \& Beaumont, 2002; Beaumont \& Rannala, 2004). Coalescent theory seeks to

145 describe the ancestral relationship of a particular gene or set of genes by recognizing that the 
146 probability of two lineages coalescing during a particular generation is inversely proportional to

147 effective population size at that time (Beaumont \& Rannala, 2004; Kuhner, 2008;). Thus, these

148 models trace separate genetic lineages back to their most recent common ancestor (Kuhner,

149 2008) and connect these genealogies to changes in effective population size (Storz \& Beaumont,

150 2002). Models such as BEAST and Msvar adopt Bayesian Markov chain Monte Carlo (MCMC)

151 methods to explore parameter space and sample the posterior distributions of the demographic

152 parameters of interest (Beaumont, 1999; Drummond \& Rambaut, 2007). A key advantage to

153 these methods is the ability to include time as one of the estimated parameters, as opposed to

154 using fixed time points as assumptions in the model. Directly estimating time is particularly

155 important for models of historical sea lamprey demography, as previous genetic studies that

156 focused on the population's origin have been criticized for using fixed, potentially-incorrect

157 dates (Eshenroder, 2014).

158 The sea lamprey is a tractable study species for coalescent modeling of effective

159 population size fluctuations because several genetic resources are available. The entire

160 mitochondrial genome is sequenced (Lee \& Kocher, 1995), enabling researchers to sequence

161 hypervariable regions of mitochondrial DNA (mtDNA). For decades, mtDNA has been widely

162 used to infer demographic processes because of its maternal inheritance, small effective

163 population size, and relatively fast rate of evolution (Avise, 1994; White et al., 2008). These

164 characteristics are particularly useful for demographic studies because uniparental inheritance

165 can be modeled without the complications of recombination, and signatures of relatively recent

166 demographic events are more readily detectable when effective population size is small and

167 mutation rate is elevated. Also, P. marinus has two non-coding regions in the mitochondrial

168 genome: Non-coding region one (NCI) is 491-bp long and non-coding region two (NCII) is 199- 
169 bp long (Lee \& Kocher, 1995). Finally, a suite of microsatellite markers has already been

170 developed for this species (Bryan et al., 2005); thus, markers from the mitochondrial and nuclear

171 genomes can be used concurrently to study the population's history. The concurrent use of two

172 genomic marker types enables intra-population replication for inferring demographic history

173 (Eytan \& Hellberg, 2010).

174 The purpose of this study was to use multiple analytical approaches and two sets of

175 genetic markers to investigate historical population fluctuations in Lake Champlain sea lamprey.

176 First, moment-based methods — including a mismatch distribution and Fu's $\mathrm{F}_{S}$ statistic — were

177 used to generate coarse estimates of historical population expansions and/or contractions based

178 on mtDNA sequence data (NCI and NCII). Second, two coalescent MCMC models were used to

179 explicitly estimate changes in effective population size over time. Mitochondrial sequence data

180 (NCII) were used to generate a Bayesian Skyline Plot (BSP) in the program BEAST to model

181 effective population size history while taking into account coalescent and phylogenetic

182 uncertainty. Previously-published allele frequency data for eight nuclear microsatellite loci

183 (Bryan et al., 2005) were also used in the program Msvar to estimate four demographic

184 parameters: historical effective population size, current effective population size, mutation rate,

185 and time. In total, we used 10 loci ( $\mathrm{n}=2$ non-coding mtdna; $\mathrm{n}=8$ nuclear microsatellites), and

186 employed both moment-based methods as well as two classes of coalescent models to explore

187 historical demography. Taken together, these data can provide insight into signatures of

188 demographic events within two separate genomes.

189

190 METHODS

191 Sample collection and mitochondrial DNA sequencing 
192 To estimate historical population fluctuations within Lake Champlain, fin tissue samples from

193 spawning-phase sea lamprey were obtained from the US Fish and Wildlife Service (USFWS) in

194 May-June 2009. All tissue collection was conducted as part of routine USFWS sampling; the

195 authors of this study received tissue, but did not handle any vertebrate specimens. First, to

196 estimate fluctuations based on mtDNA sequence data, samples were collected at three Lake

197 Champlain tributaries: Great Chazy River $(n=33)$, Malletts Creek $(n=33)$, and Beaver Brook $(n$

$198=28$ ), representing the northern, central, and southern regions of Lake Champlain, respectively.

199 A broad geographic sampling regime was used in order to test for population structure. All tissue

200 samples were stored in 95\% non-denatured ethanol and genomic DNA was extracted using

201 DNeasy Blood and Tissue Kits (Qiagen).

202 The two non-coding regions of the mtDNA genome, NCI and NCII, were amplified in all

203 Lake Champlain samples using PCR with the lamprey-specific primers CR1 (Waldman et al.,

204 2004) and LampR (5'- AATAGACGGTTGGTGGGACA - 3'). PCR reactions were performed

205 in $25 \mu$ l volumes with the following reagents: $0.2 \mu$ l Qiagen taq polymerase ( 5 units/ $\mu \mathrm{l}), 2.5 \mu \mathrm{l}$

206 10X PCR buffer (with $1.5 \mathrm{mM} \mathrm{MgCl}_{2}$ ), $10 \mu \mathrm{M}$ each primer, $10 \mu \mathrm{M} \mathrm{dNTP}$, and 50-100 ng

207 template DNA. Thermal cycler settings were set at an initial denaturation at $95^{\circ} \mathrm{C}$ for 5 minutes

208 followed by 40 cycles of $95^{\circ} \mathrm{C}$ for 45 seconds, $56.5^{\circ} \mathrm{C}$ for 45 seconds, and $72^{\circ} \mathrm{C}$ for 1 minute;

209 and a final extension at $72^{\circ} \mathrm{C}$ for 10 minutes. PCR products were cleaned using the Wizard SV

210 Gel and PCR Clean-Up Kit (Promega) and eluted in $25 \mu \mathrm{H}_{2} \mathrm{O}$.

211 To sequence the Lake Champlain samples at both non-coding regions, forward extension

212 reactions were run for each individual using the forward primers CR1 (for NCI) and CASSFN

213 (for NCII) (5’-GACCCCTAAGTTCATTGC - 3'). All primers designed specifically for this

214 study, including LampR, CR1, and CASSFN, were designed using Primer3 (Untergrasser et al., 
215 2012). Extension reactions were prepared using $1 / 4$ reactions from the BigDye Terminator v3.1

216 Cycle Sequencing Kit (Applied Biosystems). For each $20 \mu$ reaction, the following reagents

217 were combined: $2 \mu$ l Ready Reaction Mix, $3 \mu 1$ xx sequencing buffer, $3.2 \mu l$ primer $(1 \mu \mathrm{M}), 1 \mu \mathrm{l}$

218 DNA template (20-30 ng), and $10.8 \mu$ l water. Thermal cycler settings were set at an initial

219 denaturation at $96^{\circ} \mathrm{C}$ for 1 minute followed by 30 cycles of $96^{\circ} \mathrm{C}$ for 20 seconds, $50^{\circ} \mathrm{C}$ for 20

220 seconds, and $60^{\circ} \mathrm{C}$ for 4 minutes. Reactions were cleaned with $2 \mu \mathrm{l}$ of $2.2 \%$ sodium dodecyl

221 sulfate and returned to the thermal cycler at $98^{\circ} \mathrm{C}$ for 5 minutes followed by $25^{\circ} \mathrm{C}$ for 10

222 minutes. Reactions were then purified in Sephadex size-exclusion columns (GE Healthcare Life

223 Sciences) and $12 \mu 1$ of purified products were loaded into an Applied Biosystems 3130 Genetic

224 Analyzer for sequencing.

225 Sequences from individuals that successfully amplified at both non-coding regions

$226(\mathrm{n}=54)$ were concatenated into a single sequence denoted by $\mathrm{NC}_{\text {total }}$. Concatenated sequences

227 were then trimmed to a 652-bp region to exclude repetitive portions of reads prone to slippage

228 (NCI: excluded 14 bp before position 15382; NCII: excluded 28 bp after position 16173).

229 Regions prone to slippage were identified by superimposed sequences on the chromatograms.

230 All trimmed sequences were aligned with the MUSCLE algorithm (Edgar, 2004). Finally, a

231 haplotype network was generated for all $\mathrm{NC}_{\text {total }}$ haplotypes to visualize their relationship using

232 TCS v. 1.18 (Clement, Posada \& Crandall, 2000)

233 Second, to estimate population fluctuations based on nuclear microsatellite data, we used

234 previously-published allele frequency data (Bryan et al., 2005). Bryan et al. (2005) genotyped

235 individuals from Great Chazy River $(\mathrm{n}=40)$ and Lewis Creek $(\mathrm{n}=40)$ at 8 microsatellite loci.

236 There was no evidence for linkage disequilibrium or significant deviations from Hardy-

237 Weinberg equilibrium (see Bryan et al., 2005 for more details). 
239 Moment-based analyses of historical population changes

240 Historical population fluctuations were inferred using three moment-based methods. First, the

241 mismatch distribution of pairwise differences between $\mathrm{NC}_{\text {total }}$ haplotypes was plotted. The

242 observed distribution of pairwise differences was compared to the expected number of pairwise

243 differences under a model of population expansion with 1,000 bootstrap replicates in Arlequin v.

2443.1 (Excoffier, Laval \& Schneider, 2005). Second, Harpending's raggedness index (r) was used

245 to test whether the observed distribution was significantly different from the expected theoretical

246 distribution under a model of expansion (Harpending, 1994). Third, an alternative metric, Fu's $\mathrm{F}_{s}$

247 statistic, was used to test the selective neutrality of mutations ( $\mathrm{Fu}, 1997) . \mathrm{Fu}^{\prime} \mathrm{s} \mathrm{F}_{S}$ can detect an

248 excess (or deficiency) of haplotypes, given the observed haplotype diversity, thereby indicating a

249 population expansion (or contraction).

251 Coalescent analyses of historical population changes

252 Two Bayesian coalescent MCMC models were used to estimate historical demographic

253 fluctuations of P. marinus in Lake Champlain over time. First, the program BEAST v.1.6.2

254 (Drummond \& Rambaut, 2007) was used to make inferences based on NCII sequences. This

255 region was selected because of its high concentration of polymorphic sites. BEAST applies a

256 Bayesian coalescent-based procedure, using MCMC to sample the posterior distribution of

257 genealogical trees, demographic parameters over time, and coalescent events given sequence

258 information and a set of priors. For our demographic model, we applied the Bayesian Skyline

259 Plot (BSP). BSP is a change-point model that, assuming a single panmictic population, estimates

260 fluxes in population size through time and uses a smoothing procedure to visualize these changes 
261 (Drummond et al., 2005). To determine which nucleotide substitution model fit the data, Akaike

262 information criteria (AIC) values were calculated in jMODELTEST v.0.1.1 (Guindon \&

263 Gascuel, 2003). The substitution model selected by AIC was then used as a prior in BEAST. For

264 our baseline study, we assumed a strict molecular clock of $3.6 \times 10^{-8}$ substitutions per base per

265 year (i.e., 3.6\% substitutions per million years), based on previous estimates of divergence in

266 mtDNA noncoding regions in fishes (Donaldson \& Wilson, 1999). The BSP group number $(m)$

267 was set to 15 . The parameter $m$ allows adjacent coalescent intervals to be grouped so that they

268 can have the same $\mathrm{N}_{\mathrm{e}}$ - it serves to smooth the resulting BSP. The maximum effective population

269 size was set at 10,000, a high estimate based on preliminary coalescent model runs. MCMC

270 chains were run for $50^{6}$ iterations in triplicate, sampling the posterior distribution every 1,000

271 iterations. The sampling distribution of the model was evaluated in TRACER v.1.5, with the first

$27210 \%$ discarded as burn-in (Rambaut et al., 2014). Quality of the MCMC convergence was

273 assessed by the effective sample sizes (ESS): if the ESS value was less than 100, it was assumed

274 that the MCMC chain had not been run long enough to get an accurate representation of the

275 posterior distribution and the trace was discarded (Drummond et al., 2007).

276 A sensitivity analysis for the BEAST modeling was carried out on two parameters-clock

277 rate and maximum effective population size-to assess whether priors biased parameter

278 estimates. These parameters were selected because the priors were based on our preliminary

279 estimates. For each parameter change, three additional MCMC chains were run keeping all other

280 baseline input values constant. We used two alternative maximum population sizes $(20,000$ and

281 100,000) and at one alternate clock rate $\left(2.0 \times 10^{-8}\right.$ substitutions per base per year, i.e. $2.0 \%$

282 substitutions per million years). To determine how sensitive model results were to the priors,

283 runs with altered priors were compared. 

microsatellite data. Allele frequencies at eight microsatellite loci, previously published by Bryan et al. (2005), were used as input for the Bayesian MCMC model Msvar v.1.3 (Beaumont, 1999).

Msvar uses probable genealogies of allele frequency data to generate posterior probability distributions of four demographic parameters: current effective population size $\left(\mathrm{N}_{0}\right)$, historical effective population size $\left(\mathrm{N}_{1}\right)$, mutation rate $(\mu)$, and time since the demographic change began defined for each parameter to test whether the model could detect true population fluctuations 292 (Table S1). Five independent chains were run for a panmictic sea lamprey population (Bryan et al., 2005; Waldman, Grunwald \& Wirgin, 2006), under a model of exponential growth, with an average generation time of six years (Hardisty \& Potter, 1971), and a $\mathrm{N}_{\mathrm{e}} / \mathrm{N}_{\mathrm{c}}$ ratio of 0.2 (Frankham, 1995). Each chain consisted of $8 \times 10^{8}$ iterations, with sample points taken once every 10,000 iterations. The burn-in portion of the chain was excluded by truncating runs to consider only the second half. To estimate each demographic parameter, the five truncated chains were combined into one posterior distribution and peak density values were recorded for each parameter, along with 95\% highest probability density (HPD) credibility intervals. A Bayes factor was also calculated to test whether there was more support for a population expansion or contraction using the method described by Storz and Beaumont (2002). Under the Bayesian statistical framework, a Bayes factor is an appropriate metric for comparing alternative models. population contractions even with broad priors (Girod et al., 2011). These simulation models have also shown that the precision of estimates increases when demographic parameters are 
307 scaled using coalescent theory. Thus the marginal posterior distributions of the following scaled

308 parameters were also plotted: $\theta_{0}=4 \mathrm{~N}_{0} \mu$ and $\theta_{1}=4 \mathrm{~N}_{1} \mu$ (effective population sizes scaled by

309 mutation rate); $\mathrm{t}_{\mathrm{f}}=\mathrm{t} /\left(2 \mathrm{~N}_{0}\right)$ (time scaled by current effective population size).

\section{RESULTS}

312 Haplotype diversity \& population structure

313 We observed 14 unique haplotypes among the $54 \mathrm{NC}_{\text {Total }}$ mitochondrial sequences (Table 1). The

314 haplotype network of all $14 \mathrm{NC}_{\text {total }}$ haplotypes visualizes their relationships as well as their

315 relative abundance (Fig. 1). Haplotype 1 is predominant in Lake Champlain, with five other rare

316 haplotypes $(2,3,6,7$, and 9$)$ having only one base pair difference from haplotype 1 . Most of the

317 mutations within these five haplotypes are point mutations within $\mathrm{NCI}$ or the $5^{\prime}$ end of NCII

318 (Table 1). In contrast, most of the remaining rare haplotypes branch off of haplotype 7 and are

319 characterized by mutations within the A/T-rich, repetitive 3 ' region of NCII. In general, these

320 mutations in the repetitive region were retained in each subsequent repeat of the sequence,

321 leading to a larger number of overall mutations in NCII.

322 An exact test of population differentiation revealed that haplotypes were randomly

323 distributed across the three sampling locations $(\mathrm{n}=54 ; \mathrm{p}=0.265$; $\#$ dememorization steps $=$

324 10,000; \# steps Markov chain $=100,000)$. Thus, the Lake Champlain population is considered to

325 be panmictic, consistent with results from previous studies (Bryan et al., 2005; Waldman,

326 Grunwald \& Wirgin, 2006).

327

328

Moment-based analyses of historical population changes 
329 The mismatch distribution of $\mathrm{NC}_{\text {total }}$ was smooth and unimodal, suggesting that the sea lamprey

330 population has undergone an expansion (Fig. 2). The distribution of observed pairwise

331 differences closely matches the expected distribution of pairwise differences under a model of

332 population expansion, with a raggedness index that was positive, but not significant $(\mathrm{r}=0.04 ; \mathrm{n}$

$333=54 ; \mathrm{p}=0.86)$. This indicates that there was no significant deviation from the theoretical model

334 of expansion. Additionally, Fu's $F_{s}$ statistic was significantly negative $\left(F_{s}=-6.61 ; \mathrm{n}=54 ; \mathrm{p}=\right.$

335 0.02), indicating an excess of rare haplotypes, which would be predicted under a scenario of a

336 recent population expansion. Overall, these moment-based methods of studying historical

337 demography with mtDNA data strongly support a population expansion, without explicit

338 estimates of the timing and magnitude of the event.

339

340 Coalescent analyses of historical population changes

341 For the BEAST analysis of mtDNA NCII data, the best-fit model of sequence evolution

342 determined by jMODELTEST was Hasegawa, Kishino and Yano (HKY) + I, where I means

343 there is a significant proportion of invariable sites. The BSP derived from the NCII data shows a

344 decrease in effective population size starting around 400 years ago that continued until 50 to 100

345 years ago, at which point effective population size slowly began to increase (Fig. 3). The

346 sensitivity analysis showed that the maximum population size prior was directly proportional to

347 the current $\mathrm{N}_{\mathrm{e}}$ estimate; when doubled, the $\mathrm{N}_{\mathrm{e}}$ estimates also doubled. However, changes in the

348 maximum population size and clock rate priors did not affect the overall pattern of the BSP

349 (Table S2).

350 In contrast to the BEAST analysis, coalescent modeling of microsatellite alleles estimated 351 one historical population contraction. The five Msvar chains converged below a critical potential 
352 scale reduction factor of 1.2 (Brooks \& Gelman, 1998) for all four demographic parameters,

353 indicating that there was good convergence of parameter estimates. The Bayes factor (BF) for a

354 population contraction indicated very strong support for a population contraction over a

355 population expansion $(2 * \ln (\mathrm{BF})=10.3)$ (Kass \& Raftery, 1995), with the vast majority of

356 MCMC iterations estimating a population contraction (Fig. 4a). The posterior density

357 distributions reveal the estimates for each demographic parameter with 95\% HPD intervals (Fig.

$3584 \mathrm{~b}-\mathrm{d})$. There was a decrease in effective population size from approximately $2,660\left(\mathrm{HPD}_{95 \%}=\right.$

$359153,65750)$ down to approximately 50 currently $\left(\operatorname{HPD}_{95 \%}=0.06,1440\right)$ (Fig. $\left.4 \mathrm{~b}\right)$. The density

360 peak was higher, with narrower credibility intervals, for historical effective population size

361 compared to current effective population size. This population contraction was estimated to have

362 begun approximately 820 years ago, although the peak density was only $40 \%$ for this time

363 parameter, with wide credibility intervals $\left(\mathrm{HPD}_{95 \%}=0.81,71558\right)($ Fig. $4 \mathrm{c})$. Finally, there was a

364 high density peak for the mutation rate estimate, with over $80 \%$ of the estimates approaching a

365 modal value of $3.26 \times 10^{-4}$ mutations/site/generation $\left(\mathrm{HPD}_{95 \%}=3.52 \times 10^{-5}, 2.78 \times 10^{-3}\right)($ Fig.

$3664 d)$.

367 The posterior distributions of the scaled demographic parameters also indicate a

368 population contraction (Fig. 5). As predicted by simulation analyses, scaling the parameters

369 increased the precision of parameter estimates. The magnitude of the scaled population size

370 estimates were very similar to the unscaled estimates. Modal $\theta$ estimates corresponded with a

371 decline from approximately 2,335 individuals historically $\left(\mathrm{HPD}_{95 \%}=359,29614\right)$ to 60

372 individuals currently $\left(\mathrm{HPD}_{95 \%}=0.08,772\right)$. Despite increased precision, there was still some

373 overlap in the $95 \%$ credibility intervals for $\theta$ (Fig. 5A). In contrast, there was a substantial

374 increase in precision of the time estimate, suggesting a more ancient demographic event: the 
375 modal value of the scaled time distribution corresponded with an event beginning approximately

3761,230 years ago $\left(\mathrm{HPD}_{95 \%}=128,7522\right)($ Fig. $5 \mathrm{~B})$.

377

378 DISCUSSION

379 Genetic-based methods can be useful tools for studying demographic changes in the absence of

380 historical population records, though their precision is linked to the timing and magnitude of the

381 events themselves (Beaumont \& Rannala, 2004; Lawton-Rauh, 2008). In this study, we applied

382 multiple analytical approaches and used two sets of genetic markers to investigate the population

383 history of $P$. marinus in Lake Champlain. Synthesizing the results to draw conclusions about the

384 most likely historic scenario of demographic change requires a critical analysis of the results

385 generated by each method, an exploration of sea lamprey ecology, and consideration of the

386 history of land-use and management practices in the region.

387

388 Moment-based analyses of historical population changes

389 Mismatch distribution analysis is based on the assumption that demographic changes leave

390 corresponding genetic signatures in neutral sequence data and gene trees (Rogers \& Harpending,

391 1992; Harpending et al., 1998). The mismatch distribution of this study fits a model of

392 population expansion well, being both smooth and unimodal. However, a major weakness of this

393 method is its poor ability to make specific inferences about the timing of demographic changes.

394 In general, when using moment-based methods, only a general inference as to whether the

395 change was relatively recent or ancient can be made. The presence of many low-frequency

396 mutations is one indication of a 'recent' expansion (Schneider \& Exoffier, 1999). This pattern

397 was evident in the haplotype network, in which the majority of haplotypes in Lake Champlain 
398 were present in only one to three individuals (Fig. 1). Additionally, the mean number of pairwise

399 differences in the mismatch distribution can be used for a crude estimate of timing: a 'low' mean

400 can indicate a 'recent' expansion while a 'high' mean can indicate a more 'ancient' expansion

401 (Okello et al., 2005). The haplotypes in the Lake Champlain lamprey population had a mean of

4021.74 mismatches, which supports a 'recent' expansion. Yet, without explicit guidelines for

403 differentiating between 'low' and 'high', or inferring what 'recent' and 'ancient' indicate on an

404 evolutionary timescale, caution should be used in interpreting these results. Instead, these results

405 are more appropriately used as supplemental evidence to be considered alongside inferences

406 made by coalescent-based analyses.

407

408 Coalescent analyses of historical population changes

409 BEAST and Msvar analyses both detected an initial decline in effective population size. These

410 congruent results between two separate genomes strengthen the evidence for a decline; however,

411 the magnitude and timing of the event(s) remain uncertain. The BSP generated using BEAST

412 provides credibility intervals at every time point that reflect both coalescent and phylogenetic

413 uncertainty. This proved to be important, as the BSP shows that the credibility intervals for

414 effective population size ranged from 51 to 9,500. These wide credibility intervals around $\mathrm{N}_{\mathrm{e}}$

415 could be due to the limited number of segregating sites within the NCII sequence data. The

416 sensitivity analysis also showed that the maximum population size prior affected the BSP results,

417 with the estimated $\mathrm{N}_{\mathrm{e}}$ being proportional to the prior. However, the overall pattern of

418 demographic change was consistent regardless of the priors (see Table S2), indicating that the

419 estimated mean $\mathrm{N}_{\mathrm{e}}$ always showed a contraction followed by an expansion. 
421 detect the single most likely demographic trend over time based on the strongest genetic signal.

422 Bayesian models, such as Msvar, require prior distributions for each parameter (Storz \&

423 Beaumont, 2002; Beaumont \& Rannala, 2004). Generating priors can be difficult when no

424 accurate estimates of demographic parameters are available, as is the case for sea lamprey in

425 Lake Champlain. Estimates of current sea lamprey population size are poor because census data 426 are inferred from wounding rates on prey species. Moreover, no historical documentation of sea

427 lamprey population size in Lake Champlain exists. In the absence of reliable data, broad and

428 equivalent priors were used for current and historical effective population size (Goossens et al.,

429 2006). Furthermore, through simulation analyses, Girod et al. (2011) showed that Msvar is

430 powerful at detecting population fluctuations even without informed priors if the demographic

431 event was sufficiently large and ancient. The contraction detected in this analysis indicates that

432 effective population size has declined from roughly 2,660 to 50 (a low but plausible estimate

433 given the aggressive population control program and the fish's high fecundity). It is important to

434 note that these values represent best estimates based on the mode of the posterior density

435 distributions, but that the $95 \%$ credibility intervals of historical and current population size

436 overlap due to the uncertainty associated with the current effective population size parameter.

437 Scaling the population size parameters by the mutation rate did increase precision, but there was 438 still overlap in the credibility intervals. Thus, for a comprehensive population history, the Msvar 439 results must be interpreted alongside results from the BEAST and moment-based analyses.

441 Scenario 1: Evidence for fluctuations within a native population 
442 Capitalizing on multiple analytical approaches and two unique genetic data sets used in this

443 study, it is possible to infer multiple demographic fluctuations. While the mismatch distribution

444 detected a population expansion and coalescent modeling using Msvar detected a decline, the

445 results may not be in conflict. Instead, they may be detecting the two different demographic

446 changes that are both evident in the BSP generated by BEAST. By considering known events in

447 the ecological history of Lake Champlain and the approximate time scale estimated by each

448 method, we present the most parsimonious demographic history of a native sea lamprey

449 population in Lake Champlain.

450 The decline in effective population size detected by both coalescent models may correlate

451 with land-use changes and fishing pressures that began after the arrival of European settlers in

452 the mid- $18^{\text {th }}$ century. The BSP from BEAST indicated that the decline began approximately 400

453 years ago. Given that there is uncertainty around this time estimate, one hypothesis is that a

454 gradual decline in population size began around 1753 when a large number of European settlers

455 arrived in the area. At this time, human impact on the landscape increased with the onset of

456 large-scale agricultural and clear-cutting practices and, later, the construction of mills and dams

457 that would have limited upstream lamprey spawning migrations (Klyza \& Trombulak, 1999).

458 Moreover, the acceleration of the decline shown in the BSP correlates with the extirpation of

459 salmonids in Lake Champlain - the primary food source of parasitic-phase sea lamprey. The last

460 documentation of native Atlantic salmon in the basin was 1830, with lake trout extirpation

461 following shortly thereafter in the 1890s (Fisheries Technical Committee, 2009). Thus, it is

462 plausible that the population contraction detected by both coalescent models can be explained by

463 known ecological changes in the region, though some caution is warranted given that the scaled

464 Msvar distributions suggest that the decline was more ancient (ca. 1230 years ago). 
The mismatch distribution analysis may be detecting a more recent population expansion

466

467

468

469

470

471

472

473

474

475

476

477

478

479

480

481

482

483

484

485

486

487

associated with salmonid stocking in Lake Champlain. This recent expansion is supported by the upward trend in effective population detected in the BSP in the last 50 years. A stocking program began for both Atlantic salmon and lake trout in 1972 and now focuses on stocking the main part of the lake with yearlings (Marsden et al., 2003; Fisheries Technical Committee, 2009). The reintroduction of their primary food source could have allowed the sea lamprey population to expand.

While this demographic history is plausible given the ecological context of Lake Champlain over the last 300 years, it is important to consider whether the data represent two real population changes, or whether these changes are artifacts of the models. To address this issue, an important consideration is the difference in mutation rates between the nuclear and mitochondrial markers used in the study, as faster-evolving markers generally have a greater power to detect more recent events. While the mitochondrial genome tends to have a higher mutation rate than the nuclear genome due to the mutagenic properties of respiration by-products and the limited DNA repair mechanisms of the mitochondrial genome (White et al., 2008), nuclear microsatellites have an even higher average mutation rate due to strand slippage. Indeed, Msvar estimated a fast mutation rate for the microsatellites used in the study, with the narrow posterior distribution peaking at $3.24 \times 10^{-4}$ mutations/site/generation. Nevertheless, Msvar, a model that uses microsatellite markers exclusively, has been shown to have a bias towards detecting ancient declines in effective population size (Beaumont, 1999). Most recently, this tendency has been rigorously confirmed through simulation studies (Girod et al., 2011). Therefore, even if there were signatures of two demographic fluctuations in the population's history, Msvar, which can only identify a single event, is predicted to detect the more ancient 
488 decline. As to whether or not the mtDNA sequence data show a true signature of expansion

489 within the last 40 years, some insight can be gained from a comparison between BEAST model

490 inputs and outputs. Even though an extremely strict (i.e., slow) molecular clock was used as an

491 input in this analysis, the posterior distribution of effective population size still shows an upward

492 trend beginning approximately 50 years ago. Posterior estimates that deviate from prior

493 predictions generally indicate a strong genetic signal. Taken together, these lines of evidence

494 suggest that the population fluctuations detected are likely to be real.

495

496 Scenario 2: Evidence for a founder event in the early $20^{\text {th }}$ century

497 Differentiating between founder events resulting from an invasion versus bottlenecks within

498 native populations can be challenging, as both events are predicted to lead to a decline in

499 effective population size and a loss of genetic diversity (Nei, Maruyama \& Chakraborty, 1975).

500 Thus, an alternative interpretation of the data is that the population contraction detected in Msvar

501 represents a bottleneck immediately following an invasion from the anadromous Atlantic Coast

502 population sometime after the completion of the Champlain Barge Canal in 1916, while the

503 expansion detected in the moment-based and BEAST analyses represents the population boom in

504 the 1970s. While the timing of such an invasion falls within the bounds of the BEAST and

505 unscaled Msvar credibility intervals, both coalescent models assume a single panmictic

506 population. Therefore, if the present-day Lake Champlain population is significantly

507 differentiated from the anadromous population, the coalescent models could not accurately

508 estimate historical changes beginning in the differentiated population of origin. Indeed, Bryan et

509 al. (2005) found evidence for significant genetic structure between Lake Champlain and 
510 anadromous sea lamprey populations, and long-term vicariance for the Lake Champlain

511 population.

512 The mitochondrial haplotype network can provide additional insight into founder events:

513 the random sampling associated with a recent founder event should lead to the loss of rare

514 haplotypes. Therefore, a recent founder event is unlikely given the observed network, which

515 features a number of rare haplotypes branching off of a predominant ancestral haplotype (Fig. 1).

516 In sum, the majority of the evidence from the genetic models suggests that the population is

517 native to Lake Champlain, but uncertainty remains and a founder event associated with an

518 invasion in the early 1900 s cannot be completely excluded given the genetic data that are

519 presently available.

520

521

CONCLUSIONS AND FUTURE DIRECTIONS

522 This study builds upon previous research by modeling historical sea lamprey population

523 fluctuations in Lake Champlain. Considering both coalescent models and moment-based genetic

524 approaches, we conclude that multiple demographic events are likely to have occurred over the

525 past 300 years. Importantly, however, there is a large amount of uncertainty around these

526 estimates. While we argue that the data largely align with prior genetic studies and are most

527 consistent with the native hypothesis, the wide credibility intervals around our estimates cannot

528 exclude an alternative interpretation that a founder event occurred in the early $20^{\text {th }}$ century.

529 As such, we propose two potential lines of future research aimed at resolving the

530 residency debate. First, expanded genomic sampling could provide more accurate estimates of

531 historical population sizes within Lake Champlain, as well as the timing of divergence between

532 the Lake Champlain and Atlantic populations. Second, given the uncertain results from neutral 
533 genetic data, it would be useful to investigate genes that may be under selection, e.g., those

534 regulating Na/K-ATPase pumps in the gills. Population genetic analyses, gene expression

535 analyses, and physiological saltwater challenges could be undertaken in tandem. Together, these

536 lines of research may provide further clarity to the history of sea lamprey in Lake Champlain.

537

538 ACKNOWLEDGEMENTS

539 The authors thank U.S. Fish and Wildlife Service professionals in Essex Junction, VT for

540 providing sea lamprey tissue. We also thank Vicenta Hudziak and Livingston Burgess for

541 assistance in DNA sequencing and Jeremy Ward, Wayne Bouffard, Steve Smith, and Brad

542 Young for providing feedback. We are grateful to John Waldman, Amy Russell, and one

543 anonymous reviewer for helpful comments on the manuscript. The findings and conclusions in

544 the article are those of the authors and do not necessarily represent the views of the USFWS.

\section{REFERENCES}

546 Avise JC. 1994. Molecular Markers, Natural History and Evolution. Norwell, MA: Chapman \& 547 Hall.

548 Beaumont MA. 1999. Detecting population expansion and decline using microsatellites.

549 Genetics 153:2013-2029.

550 Beaumont MA, Rannala B. 2004. The Bayesian revolution in genetics. Nature Reviews Genetics $551 \quad 5: 251-261$.

552 Brooks SP, Gelman A. 1998. General methods for monitoring convergence of iterative 553 simulations. Journal of Computational and Graphical Statistics 7:434-455.

Bryan MB, Zalinksi D, Filcek B, Libants S, Li W, Scribner KT. 2005. Patterns of invasion and colonization of the sea lamprey (Petromyzon marinus) in North America as revealed by microsatellite genotypes. Molecular Ecology 14:757-3773.

558 Clement M, Posada D, Crandall K. 2000. TCS: a computer program to estimate gene 559 genealogies. Molecular Ecology 9:1657-1659. 
560 Cornuet JM, Luikart G. 1996. Description and power analysis of two tests for detecting recent 561 population bottlenecks from allele frequency data. Genetics 144:2001-2014.

562 Donaldson KA, Wilson Jr RR. 1999. Amphi-panamic geminates of snook (Percoidei:

563 Centropomidae) provide a calibration of the divergence rate in the mitochondrial DNA control

564 region of fishes. Molecular Phylogenetics and Evolution 13:208-213.

565 Drummond AJ, Rambaut A, Shapiro B, Pybus OG. 2005. Bayesian coalescent inference of past 566 population dynamics from molecular sequences. Molecular Biology and Evolution 22:1185-

5671192.

568

569

Drummond AJ, Rambaut A. 2007. BEAST: Bayesian evolutionary analysis by sampling

570

571 trees. BMC Evolutionary Biology 7:214.

572

Drummond AJ, Ho SYW, Rawlence N, Rambaut A. 2007. A Rough Guide to BEAST 1.4.

573 University of Auckland, New Zealand.

574

Edgar RC. 2004. MUSCLE: multiple sequence alignment with high accuracy and high throughput. Nucleic Acids Research 32:1792-1797.

575 Eshenroder RL. 2009. Comment: Mitochondrial DNA analysis indicates sea lampreys are

576 indigenous to Lake Ontario. Transactions of the American Fisheries Society 138:1178-1189.

577 Eshenroder RL. 2014. The role of the Champlain Canal and Erie Canal as putative corridors for

578 colonization of Lake Champlain and Lake Ontario by sea lampreys. Transactions of the

579 American Fisheries Society 143:634-649.

580 Excoffier L, Laval G, Schneider S. 2005. Arlequin ver. 3.1: An integrated software package for 581 population genetics data analysis. Evolutionary Bioinformatics Online 1:47-50.

582 Eytan RI, Hellberg ME. 2010. Nuclear and mitochondrial sequence data reveal and conceal 583 different demographic histories and population genetic processes in Caribbean reef fishes.

584 Evolution 64:3380-3397.

585 Fisheries Technical Committee of the Lake Champlain Fish and Wildlife Management

586 Cooperative. 2009. Strategic Plan for Lake Champlain Fisheries. Lake Champlain Fish and

587 Wildlife Management Cooperative, USFWS, Essex Junction, Vermont.

588 Frankham R. 1995. Effective population size/adult population size ratios in wildlife: a review.

589 Genetics Research 2:91-107.

590 Fu YX. 1997. Statistical tests of neutrality of mutations against population growth, hitchhiking

591 and background selection. Genetics 147:915-925.

592 Garza JC, Williamson EG. 2001. Detection of reduction in population size using data from 593 microsatellite loci. Molecular Ecology 10:305-318.

594

595 Girod C, Vitalis R, Leblois R, Fréville H. 2011. Inferring population decline and expansion from

596 microsatellite data: a simulation-based evaluation of the Msvar method. Genetics 188:165-179. 
597 Goossens B, Chikhi L, Ancrenaz M, Lackman-Ancrenaz I, Andau P, Bruford MW. 2006.

598 Genetic signature of anthropogenic population collapse in orangutans. PLoS Biology 4:e25.

599 Guindon S, Gascuel O. 2003. A simple, fast and accurate method to estimate large phylogenies

600 by maximum-likelihood. Systematic Biology 52:6965-704.

601 Guyette MQ, Loftin CS, Zydlewski J, Cunjak R. 2014. Carcass analogues provide marine 602 subsidies for macroinvertebrates and juvenile Atlantic salmon in temperate oligotrophic streams.

603 Freshwater Biology 59:392-406.

604 Hardisty MW, Potter IC, eds. 1971. The biology of lampreys, vol. 1. New York: Academic Press.

605 Harpending RC. 1994. Signature of ancient population growth in a low-resolution mitochondrial 606 DNA mismatch distribution. Human Biology 66:591-600.

607 Harpending HC, Batzer MA, Gurven M, Jorde LB, Rogers AR, Sherry ST. 1998. Genetic traces

608 of ancient demography. Proceedings of the National Academy of Sciences USA 95:1961-1967.

609 Kass RE, Raftery AE. 1995. Bayes Factors. Journal of the American Statistical Association 610 90:773-795.

611 Klyza CM, Trombulak SC. 1999. The Story of Vermont: A Natural and Cultural History.

612 Hanover: University Press of New England.

613 Kuhner MK. 2008. Coalescent genealogy samplers: windows into population history. Trends in 614 Ecology \& Evolution 24:86-93.

615 Lawton-Rauh A. 2008. Demographic processes shaping genetic variation. Current 616 Opinion in Plant Biology 11:103-109.

617

618 Lee WJ, Kocher TD. 1995. Complete sequence of a sea lamprey (Petromyzon marinus)

619 mitochondrial genome: early establishment of the vertebrate genome organization. Genetics

$620 \quad 139: 873-887$.

621 Marsden JE, Chipman BD, Nashett LJ, Anderson JK, Bouffard W, Durfey L, Gersmehl JE, 622 Schoch WF, Staats NR, Zerrenner A., 2003. Sea lamprey control in Lake Champlain. Journal 623 of Great Lakes Research 29:655-676.

624 McLaughlin RL, Marsden JE, Hayes DB. 2003. Achieving the benefits of sea lamprey control 625 while minimizing effects on nontarget species: conceptual synthesis and proposed

626 policy. Journal of Great Lakes Research 29:755-765.

627 Nei M, Maruyama T, Chakraborty R. 1975. The bottleneck effect and genetic variability in 628 populations. Evolution 29:1-10.

629 Okello JB, Nyakaana S, Masembe C, Siegismund HR, Arctander P. 2005. Mitochondrial DNA 630 variation of the common hippopotamus: evidence for a recent population expansion. Heredity 631 95:206-215. 
632 Rambaut A, Suchard MA, Xie D, Drummond AJ. 2014. Tracer v1.6. Available at

633 http://beast.bio.ed.ac.uk/Tracer (accessed 29 July 2015).

634 Rogers AR, Harpending H. 1992. Population growth makes waves in the distribution of pairwise 635 genetic differences. Molecular Biology and Evolution 9:552-569.

636 Schneider S, Excoffier L. 1999. Estimation of past demographic parameters from the distribution 637 of pairwise differences when the mutation rates vary among sites: application to human

638 mitochondrial DNA. Genetics 152:1079-1089.

639 Smith BR. 1971. Sea lampreys in the Great Lakes of North America. In: Hardisty MW, Potter IC 640 meds. The Biology of Lampreys, vol. 1. New York: Academic Press, 207-247.

641 Smith BR, Tibbles JJ. 1980. Sea Lamprey (Petromyzon marinus) in Lakes Huron, Michigan, and 642 Superior: history of invasion and control, 1936- 1978. Canadian Journal of Fisheries 643 and Aquatic Sciences 37:1780-1801.

644 Storz JF, Beaumont MA. 2002. Testing for genetic evidence of population expansion and 645 contraction: an empirical analysis of microsatellite DNA variation using a hierarchical Bayesian 646 model. Evolution 56:154-166.

647 Untergrasser A. Cutcutache I, Koressaar T, Ye J, Faircloth BC, Remm M, Rozen SG. 2012.

648 Primer3 - new capabilities and interfaces. Nucleic Acids Research 40:e115.

649 Waldman JR, Grunwald C, Roy NK, Wirgin I. 2004. Mitochondrial DNA analysis indicates sea 650 lampreys are indigenous to Lake Ontario. Transactions of the American Fisheries Society $651 \quad 133: 950-960$.

652 Waldman JR, Grunwald C, Wirgin, I. 2006. Evaluation of the native status of sea lampreys in 653 Lake Champlain based on mitochondrial DNA sequencing analysis. Transactions of the 654 American Fisheries Society 135:1076-1085.

655 Waldman JR, Daniels R, Hickerson M, Wirgin I. 2009. Mitochondrial DNA analysis indicates 656 sea lampreys are indigenous to Lake Ontario: response to comment. Transactions of the 657 American Fisheries Society 138:1190-1197.

658 Waples R. 1989. A generalized approach for estimating effective population size from temporal 659 changes in allele frequencies. Genetics 121:379-391.

660 White DJ, Wolff JN, Pierson M, Gemmell NJ. 2008. Revealing the hidden complexities of 661 mtDNA inheritance. Molecular Ecology 17:4925-4942.

662 Wright S. 1938. Size of population and breeding structure in relation to

663 evolution. Science 87:430-431. 


\section{Table $\mathbf{1}$ (on next page)}

Polymorphic sites among $14 \mathrm{mtDNA}$ haplotypes from the concatenated non-coding region sequences $\left(\mathrm{NC}_{\text {total }}\right)$, relative to the most common haplotype (first entry).

Base pair positions relative to the reference mitochondrial genome are provided (Lee and Kocher, 1995). Dots indicate no change, dashes indicate a deletion, and $G / C / A / T$ represent point mutations. 


\begin{tabular}{|c|c|c|c|c|c|c|c|c|c|c|c|c|c|}
\hline & & NCI & & & NCII & & & & & & & & \\
\hline ID & $\begin{array}{l}\text { Genbank } \\
\text { Accession \# }\end{array}$ & 15398 & $\begin{array}{l}15402- \\
15403\end{array}$ & 15403 & $\begin{array}{l}16049- \\
16050\end{array}$ & 16062 & 16112 & 16114 & 16115 & 16134 & 16139 & 16142 & 16169 \\
\hline 1 & GU459340 & G & - & $\mathrm{C}$ & $\mathrm{T}$ & $\mathrm{T}$ & $\mathrm{T}$ & $\mathrm{T}$ & $\mathrm{C}$ & $\mathrm{T}$ & $\mathrm{T}$ & $\mathrm{C}$ & $\mathrm{C}$ \\
\hline 2 & GU459341 & . & $\mathrm{C}$ & . & . & . & . & . & . & . & . & . & . \\
\hline 3 & GU459342 & . & - & . & . & - & . & . & . & . & . & . & . \\
\hline 4 & GU459343 & . & - & . & . & . & . & . & $\mathrm{T}$ & . & . & $\mathrm{T}$ & $\mathrm{T}$ \\
\hline 5 & GU459344 & . & - & . & . & . & A & . & - & . & . & $\mathrm{T}$ & $\mathrm{T}$ \\
\hline 6 & GU459345 & . & - & . & - & . & . & . & . & . & . & . & . \\
\hline 7 & GU459346 & . & - & . & . & . & . & . & . & . & . & . & $\mathrm{T}$ \\
\hline 8 & GU459347 & . & - & . & . & . & . & . & . & A & . & - & $\mathrm{T}$ \\
\hline 9 & GU459348 & A & - & . & . & . & . & . & . & . & . & . & . \\
\hline 10 & GU459349 & . & - & . & . & . & . & . & . & . & . & - & $\mathrm{T}$ \\
\hline 11 & GU459350 & & - & - & - & . & . & . & . & . & . & . & . \\
\hline 12 & GU459351 & & - & . & . & . & . & . & - & . & . & $\mathrm{T}$ & $\mathrm{T}$ \\
\hline 13 & GU459352 & & - & . & . & . & . & - & - & . & A & $\mathrm{T}$ & $\mathrm{T}$ \\
\hline 14 & GU459353 & & - & . & . & . & . & . & $\mathrm{T}$ & . & A & $\mathrm{T}$ & \\
\hline
\end{tabular}

1 


\section{1}

Haplotype network for 14 concatenated mtDNA haplotypes found in Lake Champlain, constructed using TCS v1.2.1 with 95\% parsimony.

The size of the circle is proportional to the relative abundance of the haplotype. Solid lines represent one point mutation and small, unfilled circles represent inferred haplotypes. The mutations resulting in branching off of haplotype 7 are concentrated in the repetitive region of the NCII 3' region, which has an elevated mutation rate due to strand slippage. 


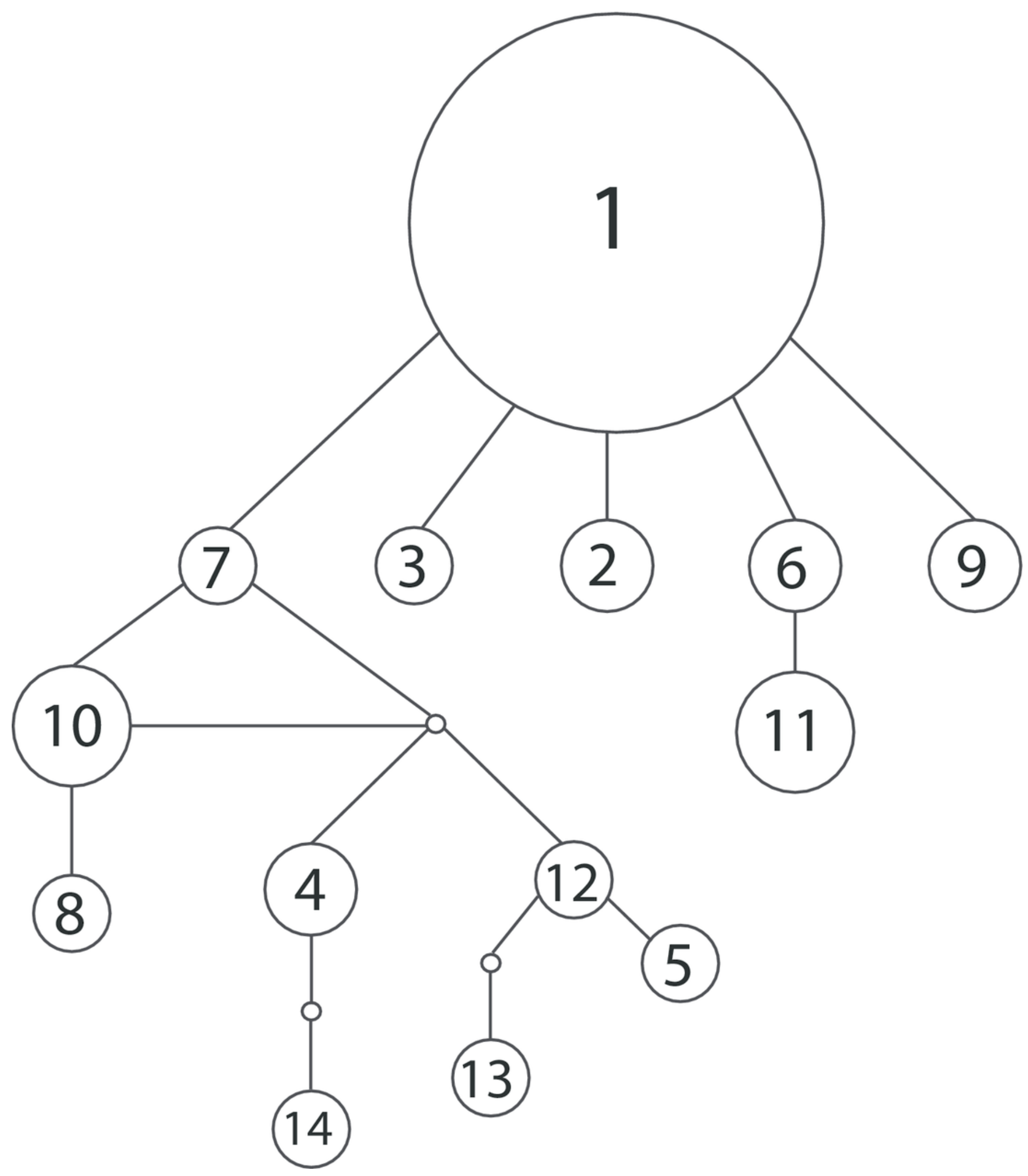


2

Mismatch distribution of the 14 concatenated mtDNA haplotypes, conducted in Arlequin v.3.1.

The black squares represent the observed number of pairwise bp differences between haplotypes, while the gray diamonds represent the expected number of pairwise bp differences between haplotypes, based on a model of population expansion (bootstrap replicates $=1,000)$. The relatively smooth and unimodal shape of the observed distribution closely matches the expected distribution for a demographic expansion.

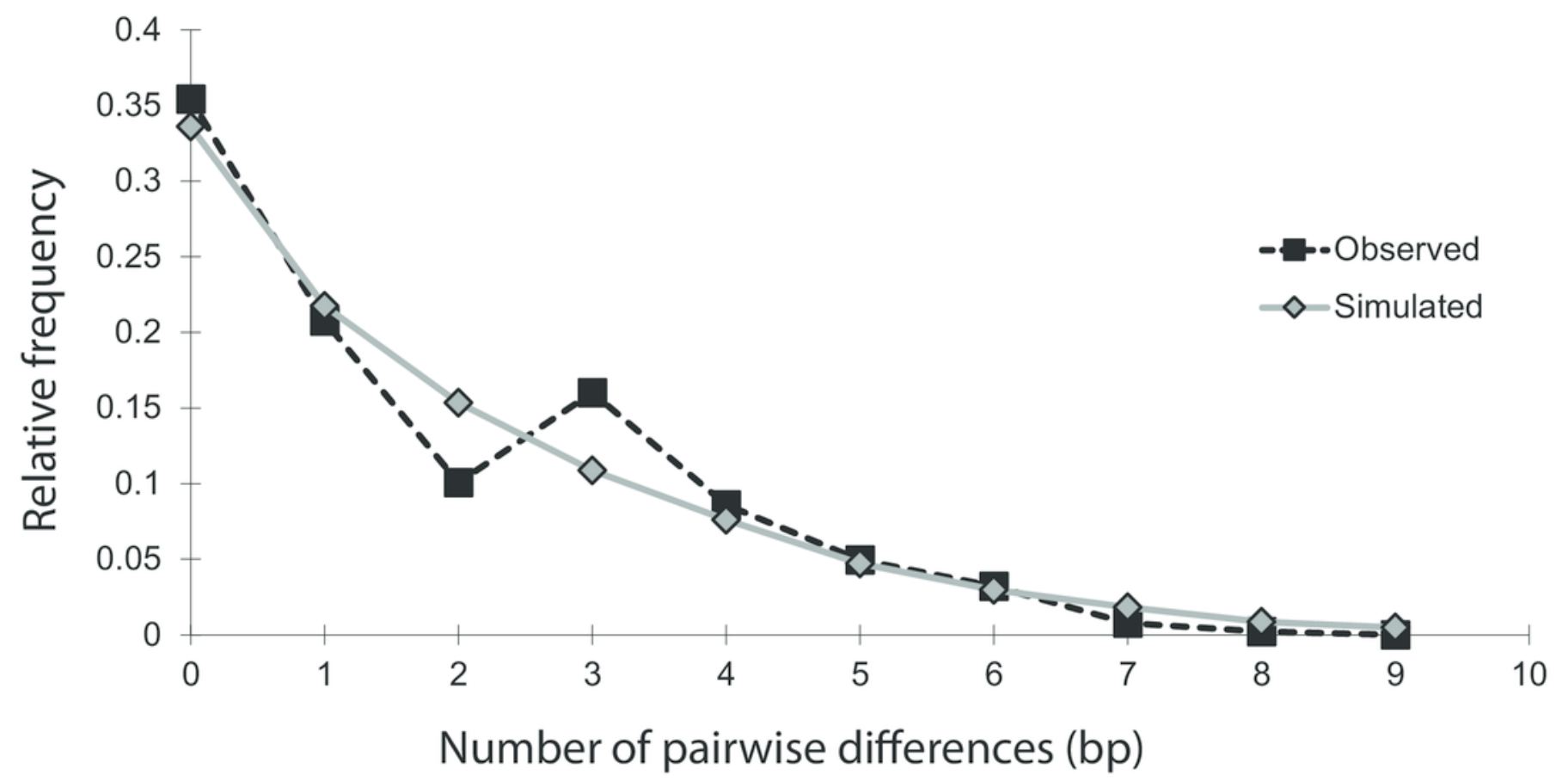


3

Bayesian skyline plot (BSP) derived from NCII sequence alignments from Lake Champlain lamprey collected in May-June 2009.
A) The mean effective population size is estimated from the Bayesian posterior distribution and is shown as the thick solid line. The horizontal dashed lines show the 95\% HPD intervals around the $\mathrm{N}_{\mathrm{e}}$ estimate. The dashed red box highlights the portion of the BSP shown in panel B; B) Zoom-in of the BSP for 400 years prior to 2009. The dashed vertical gray lines represent the timing of relevant historical events in the region. Note that the $\mathrm{x}$ and $\mathrm{y}$ axes differ between the two panels. 

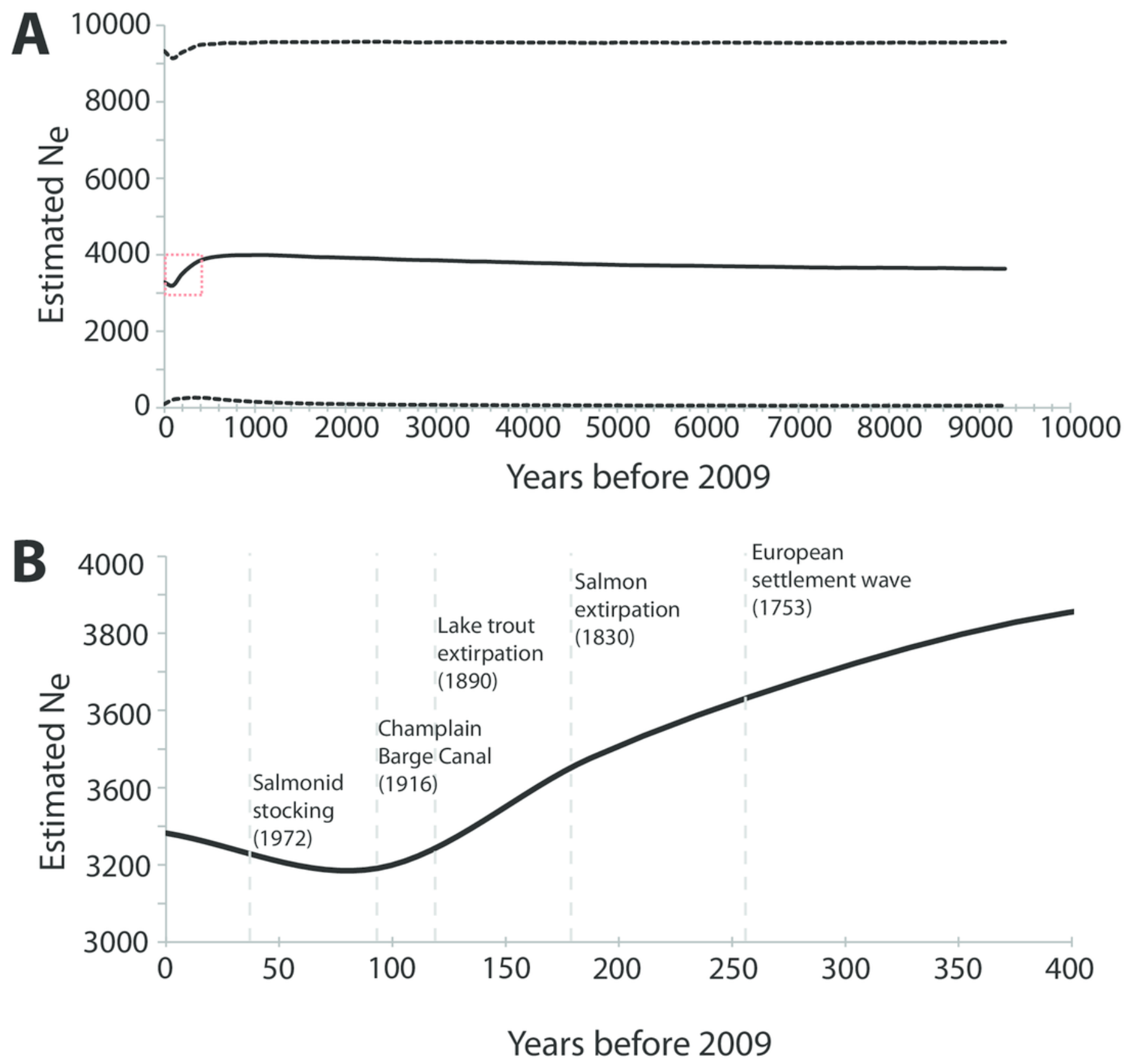


\section{4}

Msvar results based on microsatellites.

A) Density plot across MCMC iterations for $r$, which represents the ratio of current $\mathrm{N}_{e}$ to historical $\mathrm{N}_{\mathrm{e}}$, with $\mathrm{r}>1$ indicating a population expansion and $\mathrm{r}<1$ indicating a population contraction. B-D) Posterior density distributions for each demographic parameter, plotted on a $\log 10$ scale. Solid lines represent posterior density distributions and dashed lines represent prior distributions (with solid horizontal lines representing the 95\% HPD credibility intervals for the posterior distributions); B) Effective population sizes; C) Time in years since population contraction; D) Mutation rate as \# mutations/site/generation. 

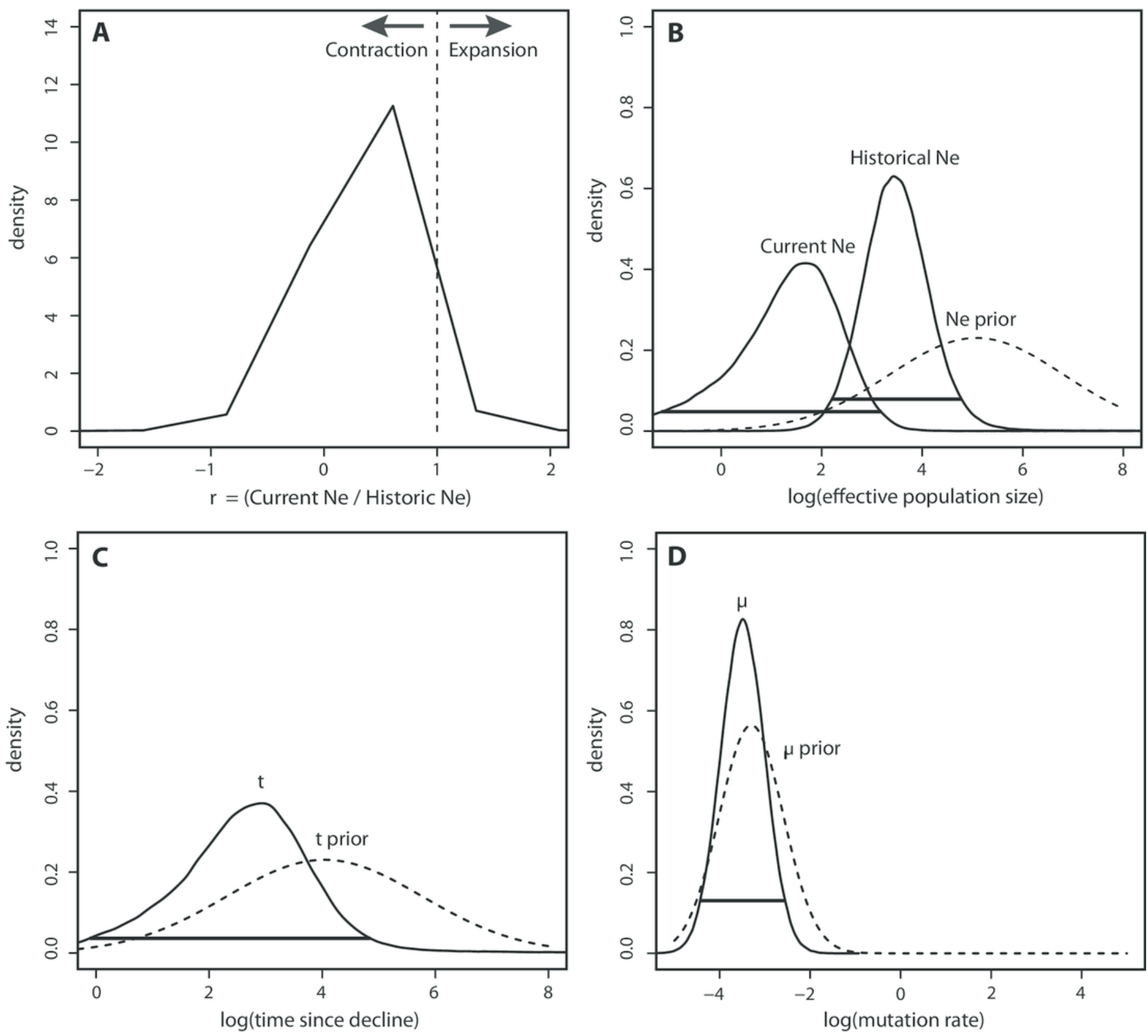


\section{5}

Marginal posterior distributions of scaled parameters from Msvar.

Solid lines represent posterior density distributions and dashed lines represent prior

distributions (with solid horizontal lines representing the 95\% HPD credibility intervals for the scaled posterior distributions). A) Scaled effective population sizes on a $\log _{10}$ scale; B) Scaled time on a $\log _{10}$ scale.
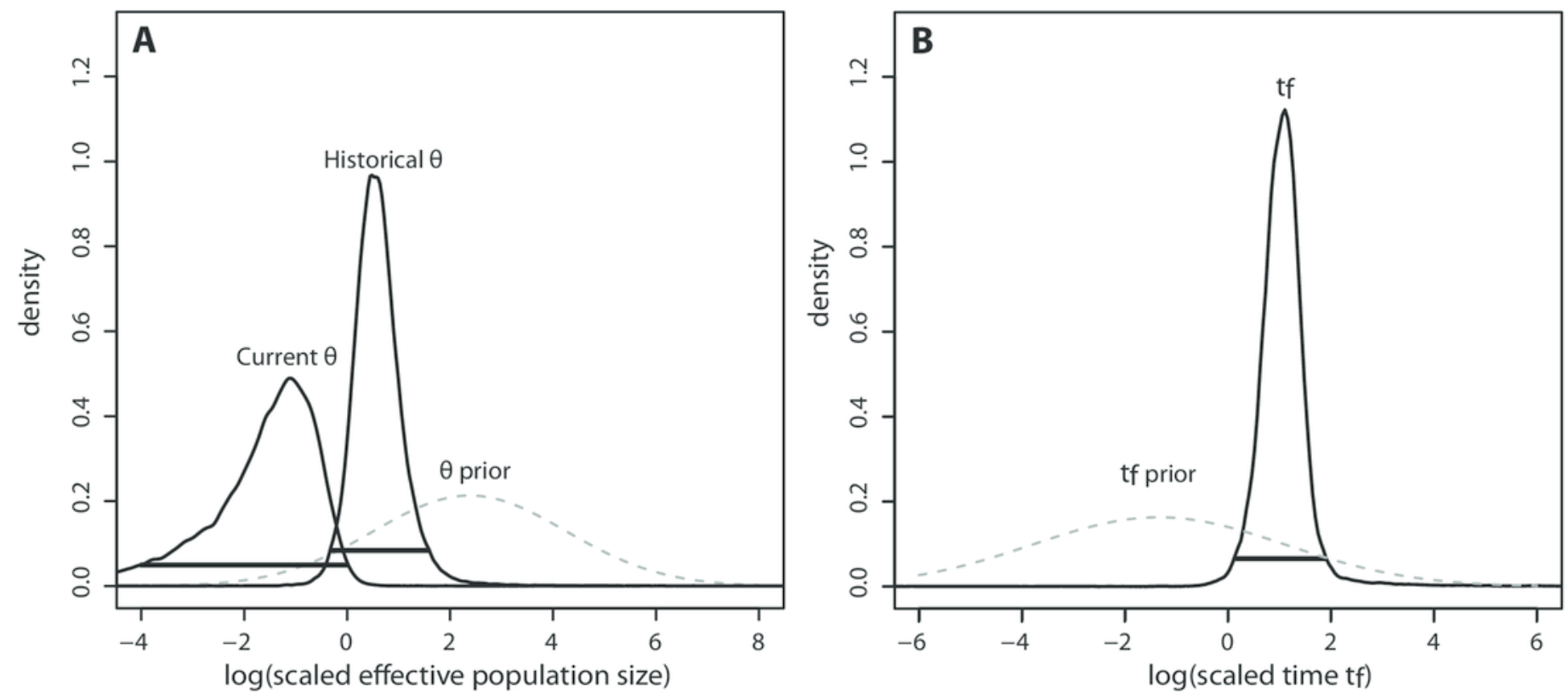